\title{
WHICH MULTIPLICATIVE SEQUENCES ARE MODIFIED CHERN CLASSES?
}

\section{ARUNAS LIULEVICIUS ${ }^{1}$}

Hirzebruch [5] has defined the notion of a multiplicative sequence: It is a natural transformation of group functors

$$
\Lambda: \tilde{K}_{c}() \rightarrow M H^{* *}(; Z),
$$

where $\tilde{K}_{c}(X)$ is the group of virtual unitary bundles over a finite cell complex $X$ [3], [4], and $M H^{* *}(X ; Z)$ is the multiplicative group of polynomials having 1 as a constant term.

An example of a multiplicative sequence is the total Chern class:

$$
C: \tilde{K}_{c}() \rightarrow M H^{* *}(; Z) .
$$

In this note we study the question: for what multiplicative sequence $\Lambda$ does there exist a natural transformation

$$
T: \tilde{K}_{c}() \rightarrow \tilde{K}_{c}()
$$

such that $T(\alpha+\beta)=T(\alpha)+T(\beta)$ for all $\alpha, \beta \in \widetilde{K}_{c}(X)$ and the following diagram is commutative:

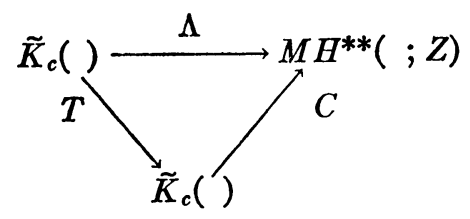

We shall call such $\Lambda$ a modified Chern class.

In general, if we consider the canonical epimorphism $\epsilon: Z \rightarrow Z_{p}$, we shall say that $\Lambda$ is a modified Chern class $\bmod p$ if there exists a $T$ such that $\epsilon_{*} \Lambda=\epsilon_{*} C \cdot T$.

1. Statement of results. Note that the set of all multiplicative sequences is a group, where $\left(\Lambda_{1}+\Lambda_{2}\right)(\alpha)=\Lambda_{1}(\alpha) \cup \Lambda_{2}(\alpha),(-\Lambda)(\alpha)$ $=\Lambda(-\alpha)=(\Lambda(\alpha))^{-1}$. This group will be denoted by $M(Z)$ if the coefficients are the integers, and $M\left(Z_{p}\right)$ if we consider $\bmod p$ coefficients, $p$ a prime.

Lemma. The modified Chern classes form a subgroup of $M(Z)$; similarly for $M\left(Z_{p}\right)$.

Received by the editors April 13, 1966.

1 The work has been partially supported by NSF grant GP-3936. 
Proof. Immediate. We denote this subgroup by $C M(Z), C M\left(Z_{p}\right)$, respectively.

Theorem 1. $C M(Z)$ is a proper subgroup of $M(Z)$. Furthermore, $C M(Z)$ is free abelian and not finitely generated.

THEOREM 2. $C M\left(Z_{p}\right)$ is a proper subgroup of $M\left(Z_{p}\right)$. Furthermore, $\operatorname{CM}\left(Z_{p}\right)$ is free abelian on $p-1$ generators.

Let $G \subset Z[[y]]$ be the multiplicative subgroup of power series in a 2-dimensional variable $y$ with constant term 1. Recall:

Theorem 3 (HIRzebruch). There is an isomorphism

$$
h: M(Z) \rightarrow G \text { such that } h(\Lambda)=\Lambda(\eta-1),
$$

where $\eta$ is the canonical line bundle over $C P^{\infty}$.

We can now describe the generators of $C M(Z)$ : let

$$
g_{n}=\prod_{j=0}^{n-1}(1+(n-j) y)^{c_{i}}, \quad \text { where, } \quad c_{j}=(-1)^{j}\left(\begin{array}{l}
n \\
j
\end{array}\right) .
$$

Theorem 4. Let $\Lambda_{n}=h^{-1}\left(g_{n}\right)$. Then $C M(Z)$ is generated by $\Lambda_{1}, \cdots$, $\Lambda_{n}, \cdots$. Furthermore, $\epsilon_{*} \Lambda_{1}, \cdots, \epsilon_{*} \Lambda_{p-1}$ form a set of generators for $\operatorname{CM}\left(Z_{p}\right)$.

Let $q$ be a natural number, $L_{q}$ the subgroup of $M(Z)$ consisting of all $\Lambda$ such that $\Lambda(\alpha)=1$ for all $\alpha \in \tilde{K}_{c}(X)$ for $X$ any complex of dimension $\leqq 2 q$. Let $C M(Z ; q)=C M(Z) / L_{q} \cap C M(Z) ; M(Z ; q)=M(Z) / L_{q}$.

Corollary 5. The cosets of $\Lambda_{1}, \cdots, \Lambda_{q}$ determine a set of generators for $\operatorname{CM}(Z ; q)$.

Corollary 6. $C M(Z ; 1) \cong M(Z ; 1), C M(Z ; 2) \cong M(Z ; 2) ; C M(Z ; 3)$ is not isomorphic to $M(Z ; 3)$.

Finally, we list the initial terms (up to degree 7) of the sequences determining $\Lambda_{i}$ :

$$
\begin{array}{ll}
g_{1}=1+y, \\
g_{2}=1 & -y^{2}+2 y^{3}-3 y^{4}-4 y^{5}-5 y^{6}+6 y^{7}, \\
g_{3}=1 & +2 y^{3}-9 y^{4}+30 y^{5}-88 y^{6}+240 y^{7}, \\
g_{4}=1 & -6 y^{4}+48 y^{5}-260 y^{6}+1200 y^{7}, \\
g_{5}=1 & +24 y^{5}-300 y^{6}+2400 y^{7}, \\
g_{6}=1 & -120 y^{6}+2160 y^{7}, \\
g_{7}=1 & +720 y^{7} .
\end{array}
$$


2. Proofs. It will be helpful to recall a proof of Theorem 3 using the splitting principle [3]. Given an $\alpha \in \widetilde{K}_{c}(X)$, realize it as a $U(n)$ bundle for some sufficiently big $h$. There is then a space $Y_{\alpha}$, a map $f: Y_{\alpha} \rightarrow X$ such that $f^{1}(\alpha)$ is a Whitney sum of line bundles and $f^{*}$ is a monomorphism in cohomology. Furthermore, if $\alpha$ and $\beta$ are two $U(n)$ bundles over $X$, then $\alpha \times \beta$ is a $U(2 n)$-bundle over $X \times X$, and we can take for the space $Y_{\alpha \times \beta}$ the space $Y_{\alpha} \times Y_{\beta}$.

Thus a multiplicative sequence $\Lambda$ is determined by its value on line bundles, hence by its value on the classifying line bundle-thus Theorem 3.

We note that we can use this argument to study additive natural transformations $T: \widetilde{K}_{c}() \rightarrow \widetilde{K}_{c}()$. Such a natural transformation will be determined, once we know its value on line bundles-we let $\gamma_{n}$ be the universal $n$-plane bundle over $X=B U(n) q$, the classifying space for $n$-plane bundles over complexes of dimensions $\leqq q$. Here $f: Y_{\gamma_{n}} \rightarrow X$ has the property that both $f^{\prime}$ and $f^{*}$ are monomorphisms.

What values can $T$ take on line bundles? This is answered by

THEOREM 7 (AdAMs [1]). Given a natural number $n$,

$$
K_{c}\left(C P^{n}\right) \cong Z[\mu] /\left(\mu^{n+1}\right),
$$

where $\mu=\eta-1, \eta$ the canonical line bundle on $C P^{n}$ with $C(\eta)=1+y$, $y$ the two-dimensional fundamental class.

Thus there are as many natural homomorphisms $T: \widetilde{K}_{c}() \rightarrow \widetilde{K}_{c}()$ as there are polynomials in $\mu$. It remains to determine $C_{0} T$ for each such $T$. To do this it is sufficient to determine $C\left(\mu^{n}\right)$ for each $n$. Now

$$
\mu^{n}=(\eta-1)^{n}=\sum_{j=0}^{n}(-1)^{j}\left(\begin{array}{l}
n \\
j
\end{array}\right) \eta^{n-j},
$$

hence

$$
C\left(\mu^{n}\right)=\prod_{j=0}^{n} C\left(\eta^{n-j}\right)(-1)^{j}\left(\begin{array}{l}
n \\
j
\end{array}\right),
$$

but $C\left(\eta^{k}\right)=1+k \eta$, hence

$$
C\left(\mu^{n}\right)=\prod_{j=0}^{n-1}(1+(n-j) y)^{c_{j}}, \quad c_{j}=(-1)^{j}\left(\begin{array}{l}
n \\
j
\end{array}\right),
$$

which is precisely the power series $g_{n}$ in $\$ 1$. This proves the first part of Theorem 4 . The second part follows if we note that if $n=\sum_{i=0}^{n} a_{i} p^{i}$, $0 \leqq a_{i}<p$, then $C\left(\mu^{n}\right)=C\left(\mu^{a_{0}}\right)$.

We wish to see what the power series $C\left(\mu^{n}\right)$ look like. If we apply the Chern character [4] to $\mu^{n}$, we know that its leading term is $y^{n}$. 
Since the filtration [1] of $\mu^{n}$ is at least $n$, we know that the Chern classes $c_{1}, \cdots, c_{n-1}$ of $\mu^{n}$ all vanish, hence

$$
y^{n}=c h_{n}\left(\mu^{n}\right)=\frac{(-1)^{n} c_{n}(n-1)}{n !}=(-1)^{n} \frac{c_{n}}{(n-1) !}
$$

by Newton's formula. Thus $C\left(\mu^{n}\right)=1+(-1)^{n}(n-1) ! y^{n}+\cdots$ higher terms.

This indicates a way of finding out whether a given series

$$
Q(y)=1+a_{1} y+a_{2} y^{2}+\cdots
$$

determines a multiplicative sequence which agrees with a modified Chern class through any finite dimensional $k-$ we do this by stripping off a coefficient at a time.

We notice that since $C(\mu)=1+y, C\left(\mu^{2}\right)=1-y^{2}+$ higher terms, that $Q(y)$ always agrees with a modified Chern class in dimensions $\leqq 2$ (these are the first two assertions of Corollary 6). We wish to exhibit a $Q(y)$ which fails to agree with a modified Chern class in dimension 3. The simplest such example is

$$
Q(y)=1+y^{3}
$$

for if $Q(y)=\prod_{n=1} C\left(\mu^{n}\right)^{b_{n}}$, then $b_{1}=b_{2}=0, b_{3} \neq 0$, but then the leading term must have coefficient divisible by 2 .

EXAMPLE.

$$
\begin{aligned}
Q(y) & =1+y+y^{2}+y^{3}+2 y^{4}+\cdots, \\
Q(y)(1+y)^{-1} & =1 \quad+y^{2}+2 y^{4}+\cdots, \\
Q(y)(1+y)^{-1} C\left(\mu^{2}\right)^{1} & =1 \\
Q(y)(1+y)^{-1} C\left(\mu^{2}\right)^{1} C\left(\mu^{3}\right)^{-1} & =1
\end{aligned}
$$

Therefore $Q(y)=1+y+y^{2}+y^{3}+2 y^{4}+\cdots$ agrees with $\Lambda_{1}^{1}+\Lambda_{2}^{-1}+\Lambda_{3}^{1}$ through dimension 3 , but disagrees with every modified Chern sequence in dimension 4.

Theorem 1 now follows, because there are no relations on the sequences $g_{n}$ in $G \subset Z[[y]]$. Theorem 2 is proved by remarking that $G_{p} \subset Z_{p}[[y]]$ is not finitely generated.

REMARK. The referee points out that

$$
g_{n}^{\prime}=1+n y
$$

are also generators of $C M(Z)$. They correspond to $\eta^{n}-1$ whereas the $g_{n}$ belong to $(\eta-1)^{n}$. The generators $g_{n}$ are used for filtration purposes and are of interest for Corollaries 5 and 6 . The $g_{n}^{\prime}$ are related to Adams' operations $\psi_{n}$. 


\section{REFERENCES}

1. J. F. Adams, Vector fields on spheres, Ann. of Math. 75 (1962), 603-632.

2. J. F. Adams and G. Walker, On complex Stiefel manifolds, Proc. Cambridge Philos. Soc. 61 (1965), 81-103.

3. M. F. Atiyah (notes by D. W. Anderson), K-theory, Harvard Univ., Cambridge, Mass., 1964.

4. M. F. Atiyah and F. Hirzebruch, Vector bundles and homogeneous spaces, Proc. Sympos. Pure Math., Vol 3, pp. 7-38, Amer. Math. Soc., Providence, R. I., 1961.

5. F. Hirzebruch, Neue Topologische Methoden in der Algebraischen Geometrie, Springer-Verlag, Berlin, 1962.

UNiversity OF Chicago 